\title{
Comunicación para la igualdad en el nuevo EEES: fundamentación crítica para el cambio social ${ }^{1}$
}

\author{
María José GÁmez FuENTES \\ Universitat Jaume I / IUDESP (Castellón) \\ gamezf@uji.es \\ Eloísa Nos ALdÁs \\ Universitat Jaume I / IUDESP (Castellón) \\ aldas@uji.es
}

Recibido: $10 / 07 / 2012$

Aceptado: 23/10/2012

\begin{abstract}
Resumen
Este estudio analiza y fundamenta una propuesta pedagógica para la integración de la docencia en igualdad en los grados de Ciencias de la Comunicación en el nuevo EEES. Para ello, realiza una revisión documental de fuentes primarias y secundarias sobre las directrices que marca este nuevo espacio en la materia objeto de estudio y las pone en discusión, por un lado, con los últimos desarrollos en teoría y pedagogía crítica de la comunicación procedentes de los Estudios Culturales, los Estudios de Género y las investigaciones para la Paz y, por otro, con las últimas tendencias en investigación en comunicación para el cambio social. Este enfoque interdisciplinar vincula el aprendizaje universitario de los futuros profesionales de la comunicación (publicitaria, audiovisual y periodística) con la incidencia cultural de sus mensajes en la igualdad en los escenarios de comunicación actuales.
\end{abstract}

Palabras clave: Comunicación, igualdad, docencia, cambio social, Bolonia

\section{Communication for the equality in the new EEES: critical foundation for the social change}

\begin{abstract}
This paper analyzes and supports a pedagogical proposal addressed to including teaching on equality in the degrees of Communication Sciences in the framework of the new EHEA. This research reviews the primary and secondary sources that define the guidelines for teaching equality in this new education arena and discusses them, on the one hand, from the focus of the most recent proposals on critical theory and critical pedagogy from Cultural Studies, Gender Studies and Peace Research, and, on the other hand, from the last trends of research on Communication for Social Change. This interdisciplinary approach links the university learning of future advertising, media and journalistic communication professionals with the cultural consequences of their messages on equality in present day communication scenarios.
\end{abstract}

Keywords: Communication, equality, teaching, social change, Bologna

\section{Referencia normalizada}

GÁMEZ FUENTES, María José y NOS ALDÁS, Eloísa (2012): "Comunicación para la igualdad en el nuevo EEES: fundamentación crítica para el cambio social”. Estudios sobre el mensaje periodístico. Vol. 18, núm. especial octubre, págs.: 325-335. Madrid, Servicio de Publicaciones de la Universidad Complutense.

${ }^{1}$ Este artículo forma parte del proyecto de investigación del Ministerio de Economía y competitividad del que ambas investigadoras forman parte: "Evaluación e Indicadores de Sensibilidad Moral en la Comunicación Actual de los Movimientos Sociales" (CSO2012-34066). La contribución de Gámez Fuentes a este trabajo ha sido realizada gracias a la estancia de investigación en la Universidad de Roehampton (Reino Unido) del 1 de junio al 31 de agosto de 2012. Dicha estancia ha sido financiada a través de la subvención concedida por la Secretaría General de Universidades del Ministerio de Educación (referencia PR2011-0448). 
Sumario: 1. Introducción. 2. Metodología. 3. Desarrollo; 3.1. Los desafíos de Bolonia; 3.2. Marco jurídico y social; 3.2.1. Igualdad y universidad; 3.2.2. Igualdad y responsabilidad de los medios; 3.3.3. Igualdad y lucha internacional contra la violencia y por una cultura de paz; 3.3. Hacia una revisión de los fundamentos y las metodologías. 4. Conclusiones. 5. Referencias bibliográficas.

\section{Introducción}

Abordar la docencia de la igualdad en la formación universitaria en Ciencias de la Comunicación (Periodismo, Comunicación Audiovisual y Publicidad y Relaciones Públicas) a raíz del nuevo escenario propiciado por las reformas de Bolonia, reclama en la actualidad una investigación interdisciplinar y crítica desde las últimas tendencias en Comunicación para el Cambio Social (Servaes, 2012) en diálogo con las aportaciones de los Estudios de Género, los Estudios Culturales y las investigaciones para la Paz.

La investigación que aquí planteamos delimita la comunicación en aras de la igualdad no solo como área de trabajo en las Ciencias de la Comunicación y en el ámbito profesional, sino sobre todo como materia (con sus contenidos, competencias y actitudes) en los planes de estudio de los actuales grados en Comunicación, una propuesta minoritaria dentro de la formación universitaria en el estado español (Gámez Fuentes y Nos Aldás, 2012).

Este trabajo profundiza, por tanto, en el marco conceptual y metodológico de esta responsabilidad de la formación universitaria en comunicación, enfocada desde la transformación socio-cultural hacia ciudadanías igualitarias, críticas y participativas, lo que reclama un proyecto educativo crítico y dialógico que pretendemos fundamentar en las siguientes páginas.

Esto no es posible sin una revisión previa del estado de la cuestión jurídico y social, que nos sitúa en un escenario en el que los parámetros de Bolonia para el EEES definen el proceso de enseñanza-aprendizaje desde la adquisición de competencias por parte del estudiantado. A esto se unen la formalización de los Objetivos del Milenio y las recomendaciones de las Naciones Unidas en materia de derechos humanos y medios de comunicación, en general, y la Ley de Igualdad, la Ley contra la Violencia de Género y la Ley de fomento de la Educación y la Cultura de Paz, en particular.

Estas iniciativas instan, en definitiva, a una renovación productiva de los medios y de la docencia universitaria que arrancan en una profunda revisión epistemológica planteada desde la teoría y la pedagogía crítica y la comunicación transformadora (Giroux et al, 1996; Giroux, 1997; Hernández, 1997; Hooks, 2010). Es por ello que fundamentaremos nuestra propuesta epistemológica sobre una metodología crítica y participativa de empoderamiento comunicativo en igualdad, que capacite al alumnado a producir productos en los que tengan protagonismo, por una parte, las mujeres y otros colectivos, invisibilizados históricamente como sujetos de cambio y transformación con respecto a los marcos de reconocimiento establecidos, y, por otra, los desajustes y articulaciones entre discursos hegemónicos, relatos mediáticos y sujetos en el marco global.

\section{Metodología}

Para llevar a cabo nuestro trabajo recurriremos, en primer lugar, a una revisión documental de las fuentes primarias y secundarias que se ocupan de la enseñanza de la 
igualdad en el EEES y en España presentando los claroscuros de este debate. Posteriormente revisaremos las posibilidades y desafíos que encierra dicho debate vinculándolo con las aportaciones epistemológicas y metodológicas que se pueden extraer de los últimos avances sobre el papel de la comunicación para el cambio social y el empoderamiento de la ciudadanía. Así, nuestra aportación constituye una revisión conceptual que pretende servir de marco epistemológico para el diseño, desarrollo, implementación y evaluación de propuestas didácticas cuyo objetivo sea la capacitación en igualdad del alumnado de Ciencias de la Comunicación.

\section{Desarrollo}

\subsection{Los desafíos de Bolonia}

La preocupación por la inclusión de la igualdad en la formación universitaria de los/as ciudadanos/as europeos/as se ha manifestado desde 1998 a través de las diferentes European Conferences on Gender Equality in Higher Education ${ }^{2}$. Pero no es hasta 2005 , con la $5^{\mathrm{a}}$ edición de dicho congreso, que se aborda el debate sobre las posibilidades que encierra Bolonia para la enseñanza de la igualdad. El nuevo EEES se considera una brecha que abre posibilidades y riesgos a la introducción de contenidos y competencias relacionados con la igualdad (Kriszio, 2008). En efecto, el comunicado de Berlín de 2003 establece ya la necesidad de incrementar la competitividad a través del fortalecimiento de la cohesión social y la reducción de las desigualdades de género a nivel nacional y europeo (Weber, 2008). Y, aunque en los siguientes comunicados de Bergen 2005 y de Londres 2007, sin embargo, no aparece, esta ausencia se balancea con una referencia esencial a la importancia de la dimensión social de la educación superior y a que la universidad refleje la diversidad de los pueblos. Es desde esa incidencia en la dimensión social de las nuevas reformas desde donde se desarrollarán iniciativas de integración de la igualdad en la formación universitaria.

Los resultados de este proceso han sido y son ampliamente cuestionados, particularmente, como aduce Ariño (2010), porque:

"El abandono del concepto de igualdad y la introducción, en este contexto, del término diversidad no deja de ser significativa, pues recoge una temática propia de las filosofías postmodernas, hegemónica en las últimas décadas. Esta deriva hacia la problemática del reconocimiento y el abandono de la perspectiva clásica de la igualdad de oportunidades y del Estado de bienestar basado en la lógica redistributiva, resultan muy significativos. Asumir que la base estudiantil universitaria debe reflejar la diversidad social presupone que dicha diversidad es positiva (o al menos aceptable) y no es resultado de la desigualdad y erradicable" (Ariño, 2010: 6).

2 Desde 1998 han tenido lugar siete congresos, a saber: 1998 (Helsinki), 2000 (Zürich), Genoa (2003), Oxford (2005), Berlin (2007), Stockholm (2009), Bergen (2012). El próximo se celebrará en Vienna en 2014. Para más información: http://www.uib.no/gender2012 y http://www.uib.no/gender2012/about/european-conferences-on-gender-equality-in-highereducation. 
Dicha crítica ha de ser tenida en cuenta e integrada dentro del diseño de un aprendizaje en comunicación para la igualdad ya que se ha de capacitar al alumnado para poder detectar las complejidades que encierra el (igualmente celebrado y cuestionado -Díez Medrano, 2010) consumo mediático de la diversidad. A su vez, los posibles resultados están fuertemente vinculados a marcos nacionales, autonómicos y universitarios particulares, y su alcance todavía está siendo debatidos (Grenz et al., 2008; Grünberg, 2011; Kortendiek, 2011), pero la fisura abierta conlleva oportunidades y desafíos para la innovación en comunicación para el cambio social.

En efecto, la oportunidad que entraña Bolonia para la capacitación en igualdad no ha sido materializada, por ejemplo, de forma homogénea en todo el territorio español, como ya abordamos en Gámez Fuentes y Nos Aldás (2012). La Universitat Jaume I, desde donde se plantea esta investigación, no ha producido tampoco los mismos resultados en todas las titulaciones tampoco. Son escasos los grados que integran en dicha universidad la perspectiva de género o las enseñanzas en materia de igualdad. Solo un $10 \%$ de los nuevos grados cumple con la normativa en materia de igualdad, en concreto, los grados de Ciencias de la Comunicación. Pero, incluso en este caso, es solo a través de la inclusión de una única asignatura obligatoria: Comunicación para la Igualdad (VV.AA., 2010).

Por otro lado, son diversas las voces, como ya recogimos en Gámez Fuentes y Nos Aldás (2012), que han criticado que la reforma de Bolonia no solo no ha supuesto una mejora de la integración de contenidos de género (e igualdad) en la formación universitaria, sino que en ocasiones ha supuesto una merma en la oferta de dichos contenidos. Esto es, por otro lado, paradójico si nos detenemos en el esfuerzo normativo que sustenta la integración de dicha capacitación en la educación superior, como veremos a continuación.

\subsection{Marco jurídico y social ${ }^{3}$}

\subsubsection{Igualdad y universidad}

En primer lugar, hemos de recordar que la igualdad figura en el artículo 1.1 de la Constitución Española de 1978 como valor superior del ordenamiento jurídico, junto con la libertad, la justicia y el pluralismo político. Por otro lado, el artículo 9.2 apunta que los poderes públicos tienen la obligación de promover la igualdad y remover los obstáculos que impidan o dificulten su plenitud; asimismo, el artículo 14 reconoce la igualdad como derecho subjetivo y prohíbe cualquier discriminación por razón de sexo.

Sin embargo, no se produce un cambio significativo respecto a las políticas para la igualdad hasta 2004, con la entrada del Partido Socialista en el gobierno central y su implantación de una legislación en materia de igualdad que convierte a España en modelo para otros países (DAW, 2009: 16 y 20). Durante este período se aprueban, por un lado, la Ley Orgánica de Medidas (LOM) de protección integral contra la violen-

3 Se puede encontrar una versión anterior del marco que aquí se recoge en Gámez Fuentes y Nos Aldás (2012). 
cia de género (1/2004) y, por otro, la Ley Orgánica para la Igualdad (LOI) efectiva de mujeres y hombres (3/2007). Ambas contemplan la necesaria inclusión de la igualdad en la formación universitaria. La LOM lo establece en su artículo 4.7 y, por su parte, la LOI lo recoge en su artículo 25. De ahí que el Real Decreto 1393/2007 de enseñanzas universitarias contenga que: “[...] los planes de estudio deberán tener en cuenta que cualquier actividad profesional debe realizarse desde el respeto a los derechos fundamentales y de igualdad entre hombres y mujeres, debiendo incluirse, en los planes de estudio en los que proceda, enseñanzas relacionadas con dichos derechos".

\subsubsection{Igualdad y responsabilidad de los medios}

En lo que respecta al papel de los medios, la LOI contempla en su artículo 36 que los medios de titularidad pública "velarán por la transmisión de una imagen igualitaria, plural y no estereotipada de mujeres y hombres en la sociedad", y respecto a los medios de titularidad privada, en su artículo 39 se detalla que estos "respetarán la igualdad entre mujeres y hombres, evitando cualquier forma de discriminación". Respecto a la publicidad en particular, la LOI también específica en su artículo 41 que "[1]a publicidad que comporte una conducta discriminatoria de acuerdo con esta Ley se considerará publicidad ilícita [...]”. Y, por último, en el ámbito de la conciliación, el artículo 14.8 señala a los medios como uno de los agentes responsables del "establecimiento de medidas que aseguren [...] el fomento de la corresponsabilidad en las labores domésticas y en la atención a la familia".

Por su parte, el Plan Nacional de Sensibilización y Prevención de la Violencia de Género, surgido al amparo de la LOM, establece como uno de sus objetivos prioritarios conseguir un cambio en el modelo de relación social y para ello insta a los medios de comunicación y a los/as responsables de agencias de comunicación y publicidad a contribuir a la transformación de los estereotipos discriminatorios. Adicionalmente, la Ley General de Comunicación Audiovisual (LGCA) 7/2010 contempla como contenidos muy graves susceptibles de sanción aquellos que fomenten odio, desprecio o discriminación por razón raza, sexo, nacionalidad, etc. o utilicen la imagen de la mujer con carácter vejatorio o discriminatorio.

En este sentido, hemos de recordar que todo el entramado jurídico enarbolado cristaliza un interés social que surge en los albores de la reconstrucción democrática y que es impulsado por las organizaciones y producciones científicas feministas, pero son los casos de violencia de género los que colocan el tema de la erradicación de la desigualdad como objetivo esencial de la agenda política y mediática (Gámez Fuentes, 2009).

\subsubsection{Igualdad y lucha internacional contra la violencia y por una cultura de paz}

Estas iniciativas sociales, producción científica y legislación desarrolladas en España han de enmarcarse, también, en el trabajo que se ha realizado en pro de las mujeres y por la paz desde instancias internacionales, ya que, de lo contrario, estaríamos abordando una cuestión estructural entroncada con los derechos humanos de forma contingente. Aunque los aspectos contextuales que dan sentido al surgimiento de la necesidad de comunicación para la igualdad en España han sido desplegados más 
arriba y son relevantes, es necesario vincularlos al debate internacional sobre igualdad, solidaridad y paz para completar los matices y las dificultades que entraña el desarrollo curricular de la docencia de la igualdad en el nuevo EEES.

Ya en 1979 la ONU aprueba la Convención para la Eliminación de todas las Formas de Discriminación contra las Mujeres; años más tarde, en 1993, se aprueba la Declaración para la Eliminación de la Violencia contra las Mujeres pero, aún así, esa misma organización considera necesario en 2009 elaborar el Handbook for Legislation on Violence against Women (DAW, 2009), de aplicación en todos los países miembros, en el que se focaliza sobre la necesidad de que la ley priorice acciones de prevención y proporcione medidas dirigidas a concienciar a la ciudadanía, a sensibilizar a los medios y a incluir contenidos sobre violencia contra las mujeres y derechos de las mujeres en el diseño curricular.

Por su parte, la Unión Europea, a través de la Hoja de Ruta para la Igualdad de Género para el período 2006-2010, reconoce como área prioritaria de actuación eliminar estereotipos sexistas e insta, por tanto, a los medios de comunicación a una implicación en dicha transformación para visibilizar a las mujeres de otro modo. Durante el período 2010-2015 la propuesta de la Hoja de Ruta es contemplar como una cuestión horizontal el contribuir a fomentar roles no discriminatorios a través de la educación.

Por otro lado, la UNESCO en su Declaración y Programa de Acción sobre una Cultura de Paz (resolución 53/243 del 6 de octubre de 1999), propone en su artículo 1 "El respeto y el fomento de la igualdad de derechos y oportunidades de mujeres y hombres" y el "derecho de todas las personas a la libertad de expresión, opinión e información". Además, incide en su artículo 15 en el papel informativo y educativo de los medios de difusión para fomentar una cultura de paz. El gobierno de España se hace eco de estas consignas a través de la Ley de Fomento (LF) de la Educación y la Cultura de Paz (27/2005), la cual en su artículo 2 establece la responsabilidad del gobierno en promover en todos los niveles educativos los valores de la cultura de paz (no violencia, tolerancia, democracia, solidaridad y justicia).

\subsection{Hacia una revisión de los fundamentos y las metodologías}

El panorama descrito hasta este momento arroja una serie de variables que nos impulsan a revisar los últimos desarrollos en pedagogía y teoría críticas sobre la comunicación para el cambio social. Tenemos, por un lado, un nuevo Espacio Europeo de Educación Superior en el que se enfatiza la diversidad frente a la igualdad (a pesar de reconocer la necesidad de acabar con la desigualdad social) y donde la integración de la igualdad en la universidad está sujeta a idiosincrasias político-económicas y geográficas particulares, a pesar de existir una legislación que, en el caso español, establece claramente la necesidad de transversalizar dichos contenidos. Esto nos hace cuestionar el aparente impacto que todas las reformas y medidas legislativas en materia de igualdad y formación universitaria parecían auspiciar, lo que nos lleva a poner en tela de juicio, inspiradas por Haraway (1991), la facilidad con la que los saberes subyugados se convierten en fuerzas de normalización. Nos referimos a las políticas, trabajos y programas de estudio que, haciéndose eco de la perspectiva de género, han reducido esta a meras descripciones o denuncias de sexismo audiovisual, lo cual es ne- 
cesario pero no suficiente. De hecho, el discurso en torno a la erradicación de la desigualdad se ha visto relegado, en el mejor de los casos, al de la violencia de género.

La defensa del sujeto político, que está en la base de las luchas contra la desigualdad, se ha visto marginado frecuentemente, en el ámbito de Ciencias de la Comunicación, a favor de aproximaciones que hacen del sujeto de estudio un mero objeto estetizado. Es momento de activar, más allá de análisis estilistas de sexismo audiovisual, el conflicto incardinado en la sujeción política que configuran las representaciones en aras de una epistemología política de la visualidad (Brea, 2005). Se necesita, pues, desarrollar una metodología de alfabetización visual con perspectiva de género, cultural y de paz que trascienda el modelo textual y forme futuros/as especialistas en las alambicadas relaciones entre los artefactos audiovisuales y la legitimación identitaria (Brea, 2005). Este proyecto entraña la convergencia de los estudios sobre la imagen con los estudios sobre la construcción visual de lo social para poner de manifiesto la interrelación entre imágenes, visualidad, dispositivos, sistemas, instituciones, discursos y cuerpos. El consumo mediático se estudia desde esta perspectiva como proceso que conforma o invisibiliza sujetos políticos determinados (Butler, 1990 y 2010). Hablamos, por lo tanto, de representaciones conceptualizadas en tanto que matrices de inteligibilidad identitaria que ocultan procesos de control y disciplinamiento de género (y otras identidades) imbricados en las narrativas audiovisuales (Butler, 1990; De Lauretis, 1984).

En aras de la innovación metodológica, queremos recuperar, pues, las consignas de Butler (2004) cuando propone transformar los marcos de inteligibilidad simbólicos a través de prácticas sociales, en diálogo con la denuncia de la violencia cultural que nos proponen las investigaciones para la paz (Galtung, 1990). Para ello es necesario partir de la recuperación, en la formación de comunicación para la igualdad, del concepto de performatividad (Austin, 1976) de los discursos y de su acción comunicativa (Habermas, 1987) desde la transversalidad de todo mensaje, que suma o resta al trabajo global por la igualdad en los diferentes planos locales y globales de cada emisor, cada cultura, cada temática.

En concreto, la metodología que proponemos giraría en torno al desarrollo de la competencia de sensibilidad moral (Pinazo y Nos Aldás, 2012) en el estudiantado, entendida desde un enfoque de pedagogía política comunicativa que dialoga con los estudios sobre indignación y esperanza (Freire, 1993 y 2001; Hessel, 2010) como una emoción moral activadora, como "sentimiento de responsabilidad no culpable" que lleva al/a la ciudadano/a a querer ser parte de un proyecto comunicativo colectivo (la publicity definida según Benavides, 1996 y 1997) y a participar del mismo (Nos Aldás y Pinazo, 2010: 109), a informarse y querer informarse, a empoderarse como actor político. La sensibilidad moral guía procesos comunicativos hacia acciones efectivas para el cambio social y tiene como consecuencia la recuperación de una "ilusión informativa, [...] [que vaya acompañada de una] demanda comunicativa [...]" en la que son claves "información, conocimiento y comunicación" (Alfaro 2005: 65-67).

La competencia de sensibilidad moral va ligada, por otro lado, a una capacitación tecnológica que posibilite al alumnado a gestar procesos comunicativos que reconozcan a los sujetos representados/interpelados como Otro en su dimensión ética, esto 
es, cuya lógica de interacción comunicativa inscriba la trascendencia, e inteligibilidad, del exceso que la relación con el Otro conlleva (Butler, 2005). Para ello será necesario acudir al concepto de "ethical witnessing" propuesto por Oliver (2004). Según esta autora los relatos mediáticos configuran estrategias de reconocimiento acomodaticias, es decir, la audiencia comprehende aquello que le es familiar. Frente a eso, Oliver aboga por otorgar valor ético y político al proceso espectatorial. Así, para dar testimonio ético, sería necesario en un primer momento el reconocimiento de lo que se ve (García Jiménez et al, 2008), pero, más allá de este, el discurso ha de activar los mecanismos necesarios para que el sujeto espectador pueda actualizar su responsabilidad ante lo que ve. La capacitación tecnológica, desde esta perspectiva, se entendería pues desde la posibilidad tanto de actuar de testigo de lo que se ve como de dar testimonio de lo que no se ve. Para ello, en primer lugar, habría que romper con los modos de representación que dinamizan un distanciamiento respecto a los sujetos representados (Chouliaraki, 2006; Maldonado Rivera, 2010). Más bien, en la línea del "periodismo de proximidad" defendido por Bell (citado en Fingeschou, 2011: 247), los medios deberían articular relatos cuyos protagonistas se constituyeran en sujetos agentes y soberanos provistos de agencia que reflexionan y actúan sobre su experiencia (como demuestra Fingeschou, 2011, en su análisis de la representación de víctimas en la cadena inglesa de Al Jazeera).

\section{Conclusiones}

La investigación realizada en torno a la revisión de las bases jurídicas, epistemológicas y metodológicas que han de alimentar el diseño de la docencia y capacitación en igualdad concluye que, para que estos procesos de aprendizaje sean eficaces, la formación ha de aunar una revisión epistemológica del papel de las representaciones como modos de sujeción y activación política con la innovación metodológica encaminada a potenciar el papel de los/as futuros/as profesionales como testigos éticos y, en el mejor de los casos, incómodos (Haraway, 1991; Preciado, 2005) respecto a las gramáticas de reconocimiento acomodaticias que los medios hegemónicos sostienen.

Así, a través de metodologías basadas en la sensibilidad moral y el testimonio ético, el estudiantado podrá desarrollar una mirada cuestionadora y mediadora desde la igualdad que derive en productos comunicativos que intervengan, desde los fundamentos de una comunicación para la igualdad verdaderamente empoderadora y de cambio social (Nos Aldás et al., 2012), proporcionando innovación en nuevos discursos para la igualdad. Se trata de generar productos, procesos y prácticas comunicativas que ejerzan una presión sobre lo simbólico desde lo social.

\section{Referencias bibliográficas}

ALFARO, Rosa María (2005): "Sociedades en movimiento: desafíos comunicativos a la sociedad de la información", en Javier ECHEVARRÍA et al (eds.), Solidaridad en red. Nuevas tecnologías, ciudadanía y cambio social. Bilbao, Hegoa, pp. 57-79.

ARIÑO VILLAROYA, Antonio (2010): "La dimensión social del EEES y la equidad participativa", ponencia inaugural de la I Jornada sobre la dimensión social de la educación universitaria en España (Valencia, 26-27 octubre 2010). Disponible en: 
http://www.uv.es/qualitat/dimsocial/documentos/ponencias/Arino_Dimension_social_Ponencia_Introductoria.pdf. Consultado el 20 de octubre de 2012 .

AUSTIN, John Langshaw (1976): How to do things with words. Oxford. Oxford University Press.

BENAVIDES, Juan (1997): Lenguaje Publicitario. Hacia un estudio del lenguaje en los medios. Madrid, Editorial Síntesis.

BENAVIDES, Juan (1996): "Los escenarios de la comunicación mediática. Por una vía diferente de investigación", Telos, vol. 44 (diciembre-febrero), pp. 132-141.

BREA, Jose Luis (2005): Estudios visuales. Madrid, Akal.

BUTLER, Judith (1990): Gender trouble. London, Routledge.

BUTLER, Judith (2004): Undoing gender. London, Routledge.

BUTLER, Judith (2005): Giving an account of oneself. New York, Fordham University Press.

BUTLER, Judith (2010): Frames of war. When is life grievable? London, Verso.

CHOULIARAKI, Lilie (2006): The spectatorship of suffering. London, Sage.

DAW (DIVISION FOR THE ADVANCEMENT OF WOMEN) (2009): Handbook for Legislation on Violence against Women. New York, United Nations.

DE LAURETIS, Teresa (1984): Alice doesn 't. Feminism, semiotics, cinema. Bloomington, Indiana University Press.

DÍEZ MEDRANO, Javier (2010): "Gestión de la comunicación en la sociedad del siglo XXI: Un 'arma' sutil para una globalización bajo sospecha", Revista de Comunicación SEECI, vol. 22 (julio), pp. 1-15.

FINGESCHOU, Tine Ustad (2011): "Suffering up close: The strategic construction of mediated suffering on Al Jazeera English", International Journal Communication, vol. 5, pp. 233-253.

FREIRE, Pablo (1993): Pedagogía de la esperanza: un reencuentro con la pedagogía del oprimido.Madrid, Siglo XXI.

FREIRE, Pablo (2001): Pedagogía de la indignación. Madrid, Morata.

GALTUNG, Johan (1990): "Cultural Violence”, Journal of Peace Research, vol. 27 (3), pp. 291-305.

GÁMEZ FUENTES, María José (2009): “Medios de comunicación y violencia de género: paradojas y vías de desarrollo”, en BERNARDO PANIAGUA, José María et al. (coords.), Retos de la comunicación ante la violencia de género. Marco jurídico, discurso mediático y compromiso social. Valencia, Tirant lo Blanch, pp. 139-156.

GÁMEZ FUENTES, María José y NOS ALDÁS, Eloísa (2012): “Comunicación para la Igualdad en clave de innovación educativa: la experiencia de la Universitat Jaume I de Castellón", en La comunicación en la profesión y en la universidad de hoy: contenidos, investigación, innovación y docencia. Madrid, UCM [en prensa]. 
GARCÍA JIMENEZ, Antonio et al. (2008): "Medios de comunicación, representaciones y percepciones. El caso de la mujer política", Estudios sobre el Mensaje Periodístico, vol. 14, pp. 175-196.

GIROUX, Henry A. (1997): Pedagogy and the Politics of Hope: Theory, Culture, and Schooling, A Critical Reader. Boulder, Westview Press.

GIROUX, Henry A. et al. (1996): Counternarratives. New York, Routledge.

GRENZ, Sabine, et al. (eds.) (2008): Bologna and Beyond: Perspectives on Gender and Gender Studies. Humboldt-Universität zu Berlin, Zentrum für Transdisziplinäre Geschlechterstudien. Disponible en: http://www.gender.hu-berlin.de/forschung/publikationen/gender-bulletins/texte-34/. Consultado el 20 de mayo de 2012.

GRÜNBERG, Laura (2011): “Introduction” en GRÜNBERG, Laura (ed.), Studies on Higher Education. From Gender Studies to Gender IN Studies and beyond. Bucharest: UNESCO-CEPES, pp. 7-17.

HABERMAS, Jurgen (1987): Teoría de la acción comunicativa. Madrid, Taurus.

HARAWAY, Donna (1991): Ciencia, cyborgs y mujeres: la reinvención de la naturaleza. Madrid, Cátedra.

HERNÁNDEZ, Adriana (1997): Pedagogy, Democracy, and Feminism: Rethinking the Public Sphere. New York, State University of New York Press.

HESSEL, Stéphane (2010): ;Indignaos! Un alegato contra la indiferencia y a favor de la insurrección pacífica. Barcelona, Ediciones Destino.

HOOKS, Bell (2010): Teaching Critical Thinking. Practical Wisdom. New York, Routledge.

KORTENDIEK, Beate (2011): "Supporting the Bologna Process by Gender Mainstreaming: A Model for the Integration of Gender Studies in Higher Education Curricula", en GRÜNBERG, Laura (ed.), Studies on Higher Education. From Gender Studies to Gender IN Studies and beyond. Bucharest: UNESCO-CEPES, pp. 211228.

KRISZIO, Marianne (ed.) (2008): 5th European Conference on Gender Equality in Higher Education. Results and recommendations. Berlin, Humboldt University.

MALDONADO RIVERA, Claudio Andres (2010): "Comunicación nomadológica. De la identidad a la alteridad", Revista de Comunicación Vivat Academia, vol. 113 (diciembre). Disponible en: http://www.ucm.es/info/vivataca/numeros/n113/DATOSS.htm. Consultado el 11 de noviembre de 2012.

NOS ALDÁS, Eloísa et al. (2012): "La eficacia cultural de la comunicación de las ONGD: los discursos de los movimientos sociales actuales como revisión”, CIC Cuardernos de Información y Comunicación, vol. 17. Madrid, Servicio de Publicaciones de la Universidad Complutense, pp. 209-237.

NOS ALDÁS, Eloísa y Daniel PINAZO (2010): "La incógnita de la persuasión en la comunicación de las ONGD: hacia unmodelo de publicidad culturalmente eficaz", 
Industrias da Persuassão. Covilhã (Portugal), Universidade da Beira Interior, pp. 87-122.

OLIVER, Kelly (2004): "Witnessing and testimony", Parallax, vol. 10 (1), pp. 7988.

PINAZO, Daniel y Eloísa NOS ALDÁS (2012): Versión Beta. "Developing Moral Sensitivity through Protest Scenarios in International NGDOS Communication", Communication Research. Disponible en:

http://www.iudesp.uji.es/wp-content/uploads/2012/04/Revisi\%C3\%B3n-Moral-sensitivity-Pinazo-and-Nos-aldas-2012BETA2.pdf. Consultado el 5 de noviembre de 2012.

PRECIADO, Beatriz (2005): “Saberes_vampiros@war", publicado en lengua francesa en Multitudes, vol. 20. Traducción al castellano de Norberto Gómez disponible en http://es.scribd.com/doc/52615234/Beatriz-Preciado-Saberes-Vampiros. Consultado el 11 de octubre de 2011.

SERVAES, Jan (2012): "Comunicación para el desarrollo sostenible y el cambio social. Una visión general”, CIC Cuadernos de Información y Comunicación, vol. 17, pp. 17-40.

WEBER, Regina (2008): Gender equality in the European students unions, en Bulletin Texte, vol. 34 (monográfico Bologna and Beyond: Perspectives on Gender and Gender Studies). Berlin. Centre for Transdisciplinary Gender Studies, Humboldt University. Disponible en: http://www.gender.hu-berlin.de/forschung/publikationen/gender-bulletins/texte-34/. Consultado el 20 de mayo de 2012.

VV.AA. (2010): Integración de la perspectiva de género y las enseñanzas en materia de igualdad de mujeres y hombres y no discriminación en los planes de estudio de grado de la Universitat Jaume I. Informe de situación. Castellón. Unidad de Igualdad y Fundación Isonomía, Universitat Jaume I. Disponible en: http://www.uji.es /bin/organs/ui/docs/diagnostico.pdf. Consultado el 8 de septiembre de 2012.

\section{María José GÁMEZ FUENTES}

gamezf@uji.es

Profesora Titular

Univ. Jaume I de Castellón/IUDESP-España

\section{Eloísa NOS ALDÁS}

aldas@uji.es

Profesora Titular

Univ. Jaume I de Castellón/IUDESP-España

Dpto. de Ciencias de la Comunicación

Facultad de Ciencias Humanas y Sociales

Universitat Jaume I

Avda. Sos Baynat, $\mathrm{s} / \mathrm{n}$

12071 Castellón 